\title{
Fidelity of Robotic Chemical Operations of Homogenous and Heterogeneous Reactions
}

\author{
Guoqiang Wang ${ }^{1, \S}$, Bo Xin ${ }^{2, \S}$, Gaobo Wang ${ }^{1}$, Yong Zhang ${ }^{1}$, Yunwen $\mathrm{Lu}^{1}$, Lin $\mathrm{Guo}^{1}$, Shuhua $\mathrm{Li}^{1}$, Chunlin Chen ${ }^{2, *}$,
} Xu Cheng ${ }^{1,3, *}$, Jing $\mathrm{Ma}^{1,3, *}$

${ }^{1}$ Key Laboratory of Mesoscopic Chemistry of MOE, School of Chemistry and Chemical Engineering, Nanjing University, Nanjing, 210023

${ }^{2}$ Department of Control and Systems Engineering, School of Management and Engineering, Nanjing University, Nanjing, 210093

${ }^{3}$ Jiangsu Key Laboratory of Advanced Organic Materials, School of Chemistry and Chemical Engineering, Nanjing University, Nanjing 210023

${ }^{\S}$ These authors contributed equally.

*Correspondence Authors: majing@nju.edu.cn; clchen@nju.edu.cn; chengxu@nju.edu.cn

\begin{abstract}
Chemical synthesis always involves laborious and time-consuming operations. Autonomous chemical synthesis systems can liberate experimentalists from those routine tasks, but the design of automatic chemical reaction machines is of great challenge. We describe herein the development of a real-life automatic chemical operation platform by using a coordinated multi-robot system with artificial intelligence and machine learning methods. The capacities of this platform were demonstrated by its application in condensation reaction of 2,4-dinitrophenyldrazine with formaldehyde, and automatic catalyst evaluation of a heterogeneous aza Diels-Alder reaction, suggesting the designed machine is applicable to both homogenous and heterogeneous chemical reactions. Besides, the excellent repeatability and reproducibility of robotic tests on these two model reactions indicate the great promise of massive transfer of manual to automatic operation.
\end{abstract}

\section{Keywords}


Automatic chemical systems, condensation reaction, aza Diels-Alder reaction, coordinated multi-robot systems, catalyst screening

\section{Introduction}

Automation in chemistry emerged as a highly efficient strategy to carry out operations that involve routine works. This concept demonstrated its power in solid-phase peptide synthesis. ${ }^{1}$ Recently, the development of robot technology and computing capability boost chemical automation dramatically. ${ }^{2}$ For example, organic chemistry witnesses the unprecedented involvement of automation in the reaction design by machine learning ${ }^{3}$ and data feedback, ${ }^{4}$ as well as abstraction and formalism of procedures. ${ }^{5}$ The automation also exhibited its broad coverage in the syntheses of various distinct small molecules in one platform. ${ }^{6}$ Besides the expenditure in automated synthetic study, the automation in analytic chemistry also shaped the workflow in connection with reactions at various stages..$^{7-9}$ Another great impact of automation in chemistry was well presented in the search of new materials, which required multiple dimensions of parameters to be optimized in a huge amount of optimization works. ${ }^{10-12}$

In the established ways to fulfill the automation, flow chemistry was widely adopted from laboratory scale ${ }^{13-}$ 15 to industrial productions. ${ }^{16}$ Recently, one seminal work of Burger and co-workers exhibited the breakthrough of robotic chemistry in their research on the photocatalyst for hydrogen production from water. ${ }^{17}$ By a combination of explicit robot engineering, the Al-enabled by Bayesian optimization in one system, the reaction showed the batch process in autonomous system could achieve unprecedented efficiency and duration. With all these landmark progresses, however, work as the benchmark to provide a comparison of the fidelity of results from robotic/manual chemical operation is not explored, which is necessary to test the reliability of robotic chemistry when a process shifts from personnel to autonomous devices. One example reported by Fleischer and co-workers in their study using a dual-arm robot showed the 
robot could achieve a comparable result during the pipetting liquid. ${ }^{7}$ However, a reaction to benchmark the whole effect of a robotic/manual is still required to be developed.

Although many routine and fixed chemical operations can be automated using automatic equipment or even robotics, there are great challenges for the design of automatic chemical machines. Compared with its counterpart of human beings, the intelligent machine (or robot) is good at repeated operations with high precision, but is weak in reasoning, making decision and acting with uncertainties for complex tasks, while these capabilities are desirable and necessary for an unmanned universal chemical robotic system. To be specific, to ensure the fidelity of the chemical reaction machine, the following issues need to be well tackled. 1) A single robotic arm fixed or on a mobile platform is not enough for a universal and $24 / 7$ working chemical robot, which is also much limited to the trade-off between flexibility and precision. ${ }^{18} \mathrm{~A}$ coordinated multirobot system is a better choice for such application scenarios, while the design of coordinated multi-robot systems is much more challenging than single robotic systems. ${ }^{19}$ 2) The designed robotic system needs to be universal and expandable for versatile chemical reactions. Modular design is one of the important methods for expandable implementation, while it is a great challenge for the modular design of a complex system consisting of heterogeneous subsystems. 3) For an unmanned automatic chemical reaction machine, machine learning techniques such as deep reinforcement learning ${ }^{20}$ need to be applied for the task allocation, path planning, motion control of the automatic reaction system. The automation and digitization of the whole chemical reaction process also make it possible to store all the historical data of the reactions and to make the system self-adaptive for future upgrading.

Our objective is to design an unmanned, $24 / 7$ working, universal automatic chemical reaction machine with high precision and efficiency, which consists of a mobile robot platform, a guided on-rail robotic arm and a pipetting robot. A total solution of machine learning algorithms has also been developed to drive the whole system to work autonomously and intelligently. As shown in Fig. 1a, the designed system is implemented by two cooperative robots (i.e., a wheeled mobile robot with a robotic arm for materials carrying and a guided 
on-rail robotic arm for chemical operations), a reagent station and a reaction staging with magnetically stirred reactors. The reliability of the platform was evaluated through the comparison of robotic and manual operation on two model reactions: 1) the condensation reaction of formaldehyde and 2,4-dinitrobenzene hydrazine; 2) a heterogeneous aza Diels-Alder reaction (Fig. $1 \mathrm{~b}$ and $1 \mathrm{c}$ ). It will be demonstrated that the designed automatic chemical reaction machine can perform different operation steps of multiple experiments, and exhibits excellent repeatability and reproducibility through to a fidelity test on the model reaction of 2,4-dinitrophenyldrazine and formaldehyde. ${ }^{21-23}$ Autonomous catalyst-performance testing on an "on water" aza Diels-Alder reaction ${ }^{24}$ shows the potential of this system on high-throughput reaction screening, and also suggests the adaptivity of this system in both homo- and heterogeneous reactions. The success of the designed coordinated multi-robot system for automatic chemical experiments provides a promising approach for the wide application of universal unmanned chemical reaction machine and even makes it possible for the implementation of a completely unmanned chemical laboratory by combining the latest development of artificial intelligence and robotics. ${ }^{25,26}$ 
(a)

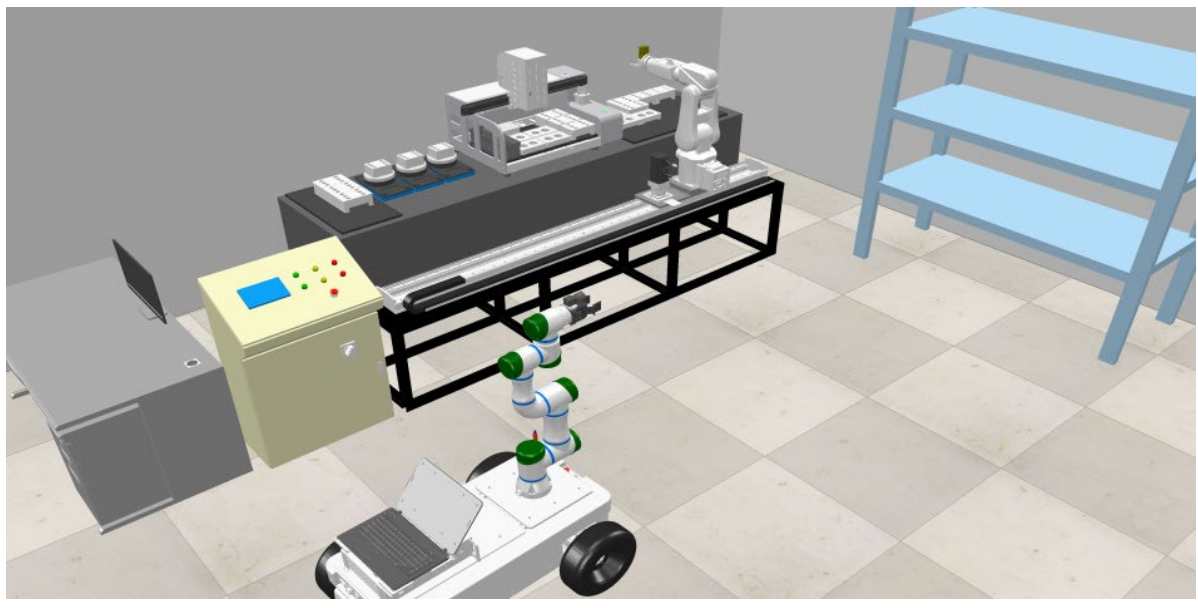

(b)

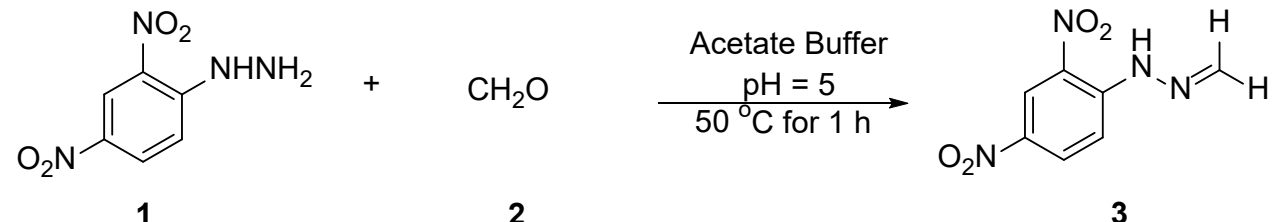

(c)

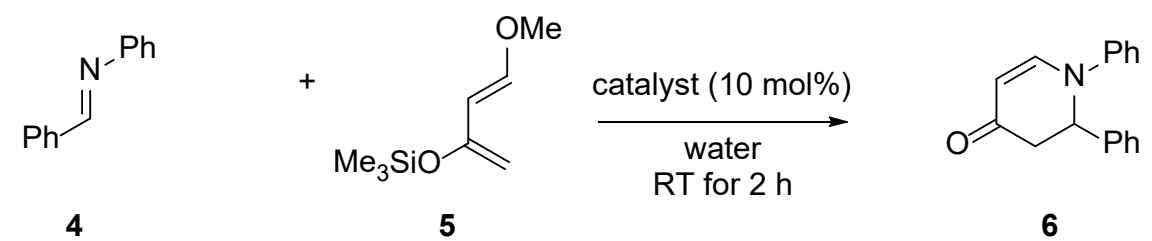

Fig. 1 (a) Autonomous chemical operation platform; (b) Model reaction I: condensation reaction between formaldehyde and 2,4-dinitrobenzene hydrazine (DNPH); (c) Model reaction II: simulation of catalyst screening for a heterogeneous aza Diels-Alder reaction.

\section{Experimental Methods}

The automation section was conducted on the operation area using the guided on-rail robot assisted with the wheeled mobile robot for materials carrying. General procedure to determine the fidelity of results from robotic/manual chemical operation robotic/manual chemical operation is described as follows. 
Procedure $\mathrm{A}$, reaction of 2,4-dinitrophenyldrazine $(\mathbf{1}, \mathrm{DNPH})$ and $\mathrm{CH}_{2} \mathrm{O}(\mathbf{2})$ : Four reactions charged with the solution of formaldehyde, DNPH and acetate buffer $(\mathrm{pH}=5)$ of different molar ratio was conducted in parallel. These reaction sets were carried out for 3 runs. For comparison, the personnel operation was performed with a manual pipette and the same tips as that used by robot. All reactions were performed at $50{ }^{\circ} \mathrm{C}$ for $60 \mathrm{~min}$, after which, several drops of the mixture were diluted with MeCN and analyzed on HPLC. More details are available in Supporting Information.

Procedure B, simulation of catalyst screening for aza Diels-Alder reaction: In a $5 \mathrm{~mL}$ reaction tube was placed $N$-benzylideneaniline $4(54.4 \mathrm{mg}, 0.30 \mathrm{mmol})$. A stock solution of a catalyst $(0.6 \mathrm{~mL}, 0.05 \mathrm{M})$ and Danishefsky's diene 5 (90 $\mathrm{mL}, 0.45 \mathrm{mmol})$ were then added successively by manual operation and our automation system, respectively. The mixture was stirred vigorously at $25^{\circ} \mathrm{C}$ for 2 hours, after which the reaction mixture was diluted with $0.5 \mathrm{~mL} \mathrm{CDCl}$, and (oxybis(methylene))dibenzene (119 $\mathrm{mg}, 0.6 \mathrm{mmol}$ ) was added as an internal standard. $100 \mu \mathrm{L}$ of the mixture was transferred to an NMR tube by pipetman and $\mathrm{CDCl}_{3}(0.3 \mathrm{~mL})$ was added. The yield was determined by ${ }^{1} \mathrm{H}$ NMR by the integration of the desired product 1,2-diphenyl-2,3dihydropyridin-4(1H)-one and internal standard. More details are available in Supporting Information.

\section{Computational Methods}

Density functional theory (DFT) calculations were performed at B3LYP-D3/Def2-TZVP//B3LYP-D3/Def2-SVP level of theory. ${ }^{27-31}$ The solvent effect was treated with the PCM solvation model ${ }^{32}$ with water as the solvent in structure optimization and single-point energy calculations (see the Supporting Information for more details).

\section{Results and Discussion}

\section{Simulation and System Design}


The designed real-life automatic chemical reaction machine is implemented by a cooperative multi-robot system with artificial intelligence and machine learning technologies, and aims at providing a totally unmanned, scalable, 24/7 working chemical reaction solution for most chemical experimental applications. The chemical experiment automation system includes two types of robot arms, i.e., on a rail and on a mobile platform, which can coordinate with each other and conduct multiple experiments at the same time.

We implement the robotic chemical operation system physically with a digital $\operatorname{twin}^{33}$ to increase the intelligence of the whole system, shown as in Fig. 2, so that we can help design the physical robotic chemical system, evaluate and test the system performance, predict future behavior, and refince the control to optimize the chemical operations. Using the digital twin, the robotic chemical system first produces the operation process in the 3D simulation environment, where multiple sets of experiments are allocated and coordinated according to the reaction substances, operation types and operating time of the relevant experiments. Then the cooperation workflow is scheduled for multiple robots, and is simulated to verify and guarantee the performance of the robotic chemical system. Finally, the verified workflow is directly transmitted to the physical robotic system through the communication protocol and is carried out to perform the corresponding physical experimental operations. At the same time, the streaming data from the physical system and its sensors are sent back to the digital twin to keep it up-to-date for future optimization, which is powered by such artificial intelligence algorithms as deep learning and reinforcement learning.

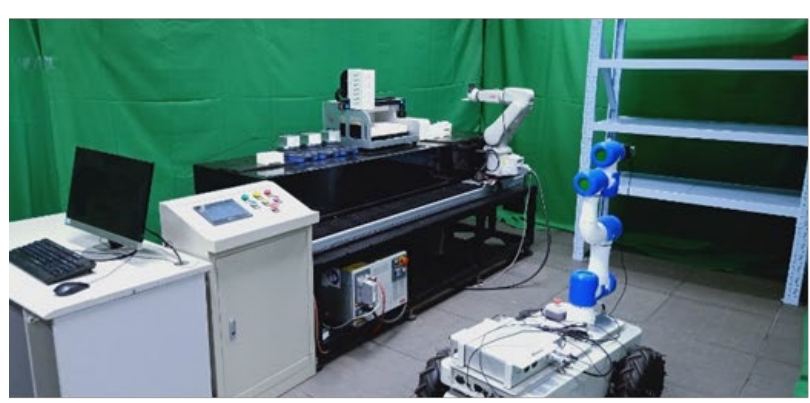

(a)

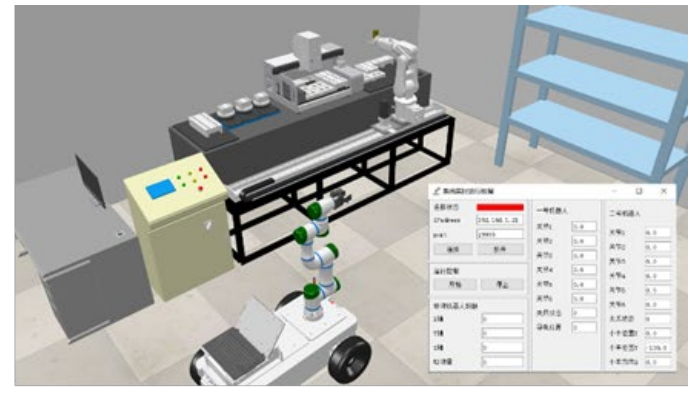

(b)

Fig. 2 Physical implementation (a) and its digital twin (b) of the automatic chemical reaction system. 
During the execution of the experiment, the wheeled mobile robot with a six-axis robotic arm for materials carrying completes the transportation of chemical reaction materials. A pipetting robot adds the reaction materials to the reaction vessel in a specific amount. The guided on-rail robotic arm (another six-axis robotic arm fixed on the linear guiding rail) can move the reaction vessel to the corresponding device for heating or stirring operations, and place the reaction vessel in the area to be tested after the reaction is completed. The testing results of experimental products can be detected and recorded, and are provided to experimental researchers or the automatic analysis algorithms for further screening and testing. The robotic chemical system can perform different operation steps of multiple experiments at the same time, and help complete most of the operations with high accuracy, which releases researchers from high-risk, tedious and high repetitive procedures, and allows the researchers to concentrate on more creative work.

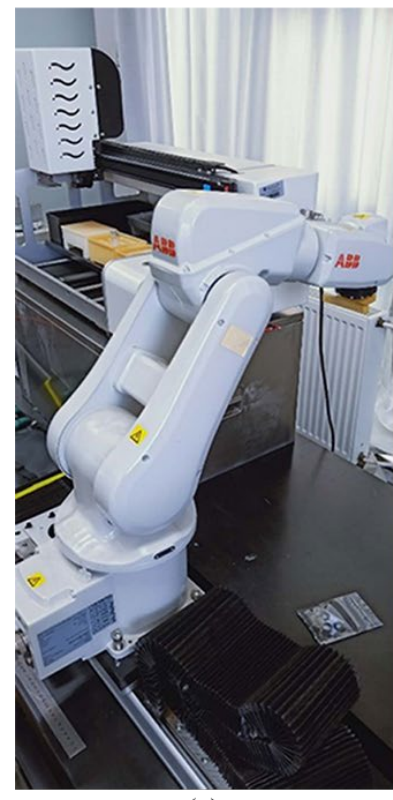

(a)

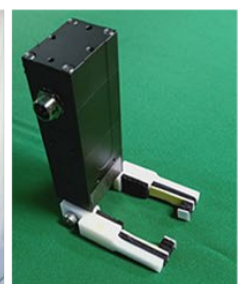

(b)

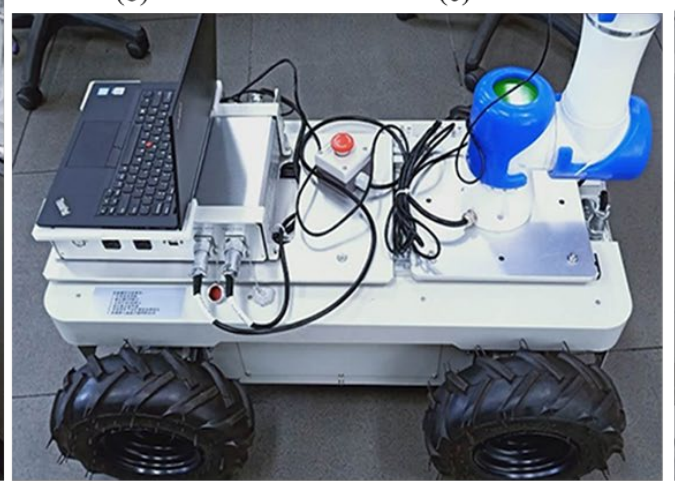

(e)

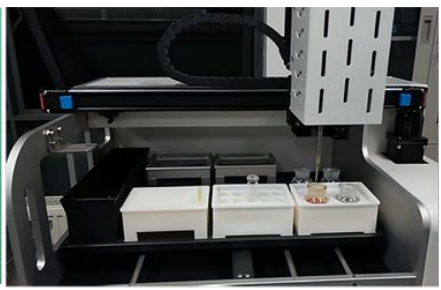

(c)

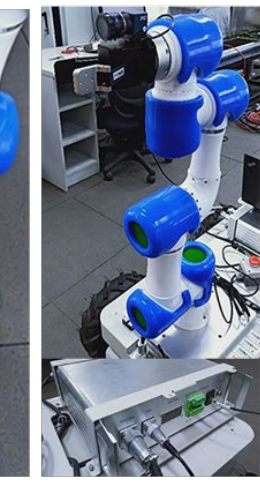

(f)

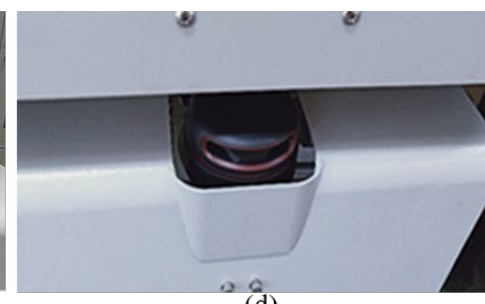

(d)

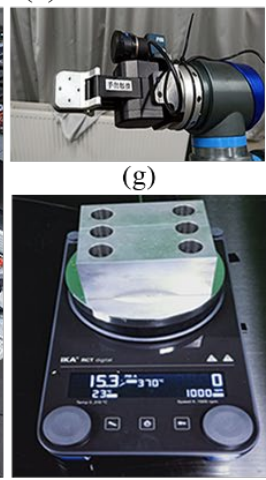

(h)

Fig. 3 Physical implementation of the robotic chemical experimental system, including (a) six-axis manipulator on a linear guide module; (b) a manipulator fixture with a parallel end; (c) pipetting workstation; (d) lidar for obstacle-avoidance and map-building; (e) trolley for reclaiming and feeding; (f) robotic arm; (g) parallel 
gripper with a non-slip elastic sponge cushion to protect the reaction vessel and an adjustable clamp; $(\mathrm{h})$ the magnetic stirrer.

As shown in Fig. 3, the physical implementation of the robotic chemical experiment system consists of three parts, i.e., a guided on-rail robotic manipulator, a pipetting robot, and a wheeled mobile robot as an AGV (autonomous guided vehicle) trolley. The guided on-rail robotic manipulator consists of a linear guide module and an ABB IRB120 six-axis manipulator (Fig. 3a). The linear guide module is driven by AC synchronous servo motors and allows the robotic manipulator to move on a fixed rack track with a control accuracy of $1 \mathrm{~mm}$, which can move the base of the manipulator quickly and smoothly to the optimal operating position. The ABB IRB120 six-axis manipulator is controlled by an IRC5 compact control box with a positioning accuracy of $\pm 0.01 \mathrm{~mm}$. The high-precision position control ensures that the robotic manipulator can move to the specified position accurately in complex environments and can accurately grasp the target object. In order to adapt to the reaction vessels with different diameters, a manipulator fixture with a parallel end (Fig. 3b) is designed for supporting multiple diameters. To guarantee the accuracy and efficiency of moving the chemical reactant, a special three-degree-of-freedom single-channel pipetting workstation (Fig. 3c) is designed and integrated into the whole system for pipetting. The precision of liquid transfer is $1 \mu \mathrm{L}$.

Equipped with a lidar (Fig. 3d) for obstacle-avoidance and map-building, the AGV trolley (Fig. 3e) uses a Japanese Tamagawa high-power motor and industrial-grade driver with encoders to ensure that the trolley moves in accordance with the target trajectory. Using differential control on the motors, the AGV trolley can turn and move more flexibly in the laboratory. In the laboratory, the AGV trolley can navigate to the designated position to complete the tasks of reclaiming and feeding. The robotic arm mounted on the AGV is selected as a collaborative robotic arm developed by "Joint Intelligence" as shown in Fig. $3 \mathrm{f}$ with a controller of STM32F407VET6. This robotic arm contains six degrees of freedom with a payload of $3 \mathrm{~kg}$ and the repeatability accuracy of $\pm 0.05 \mathrm{~mm}$. The parallel gripper at the end of the robotic arm is shown as in Fig. $3 g$. The clamping surfaces are always parallel during opening and closing, and there is a non-slip elastic sponge to 
cushion and protect the reaction vessel. The clamp can provide an adjustable clamping force of up to $20 \mathrm{~N}$ and can easily grab objects of $1 \mathrm{~kg}$. In addition, the threaded holes for fixing the clamps are designed at the ends of the parallel clamps to facilitate the expansion of applications, and the clamping tools can be extended for different chemical reaction vessels.

Besides the multi-robotic systems, there are some standard instruments for such operations as stirring and heating. As shown in Fig. 3h, the magnetic stirrer is selected as the stirring device and the robotic manipulator may add a magnet into the reaction test tube and place it on the test tube rack.

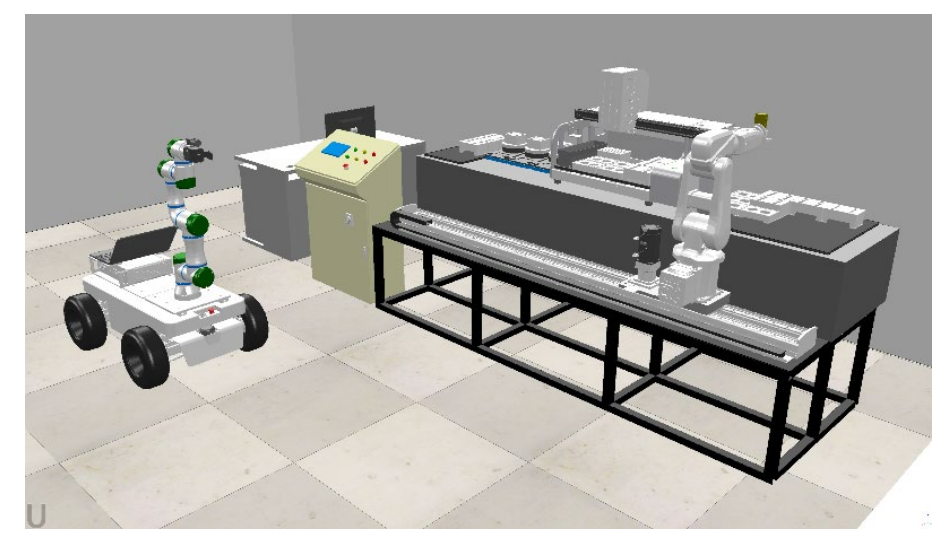

Fig.4 Screenshot of part of the simulation scene of the robotic chemical experiment system.

The software components of the robotic chemical experiment system include a simulation system for the digital twin, central control software for the physical systems and a communication system. The simulation system uses the HedraSMF software as the simulation platform. As shown in Fig. 4, the scenario is designed in the HedraSMF simulation software including the raw material area and the operation area. To run the workflow verified in the simulation on the physical operation systems, a central control software is required to coordinate the simulation system with the physical workstation and the coordinated multi-robotic system. We design the corresponding control systems for all the robots and equipments. The communication between different robots, and between the physical systems and the digital twin (the simulation system), are 
solved through TCP communication. The control and communication structure is shown as in Fig. 5. We set the central controller as the TCP server, the guided on-rail robotic manipulator as the TCP client 1, the AGV trolley with a on-board high-performance computer as the TCP client 2, and the pipetting robot as the TCP client 3.

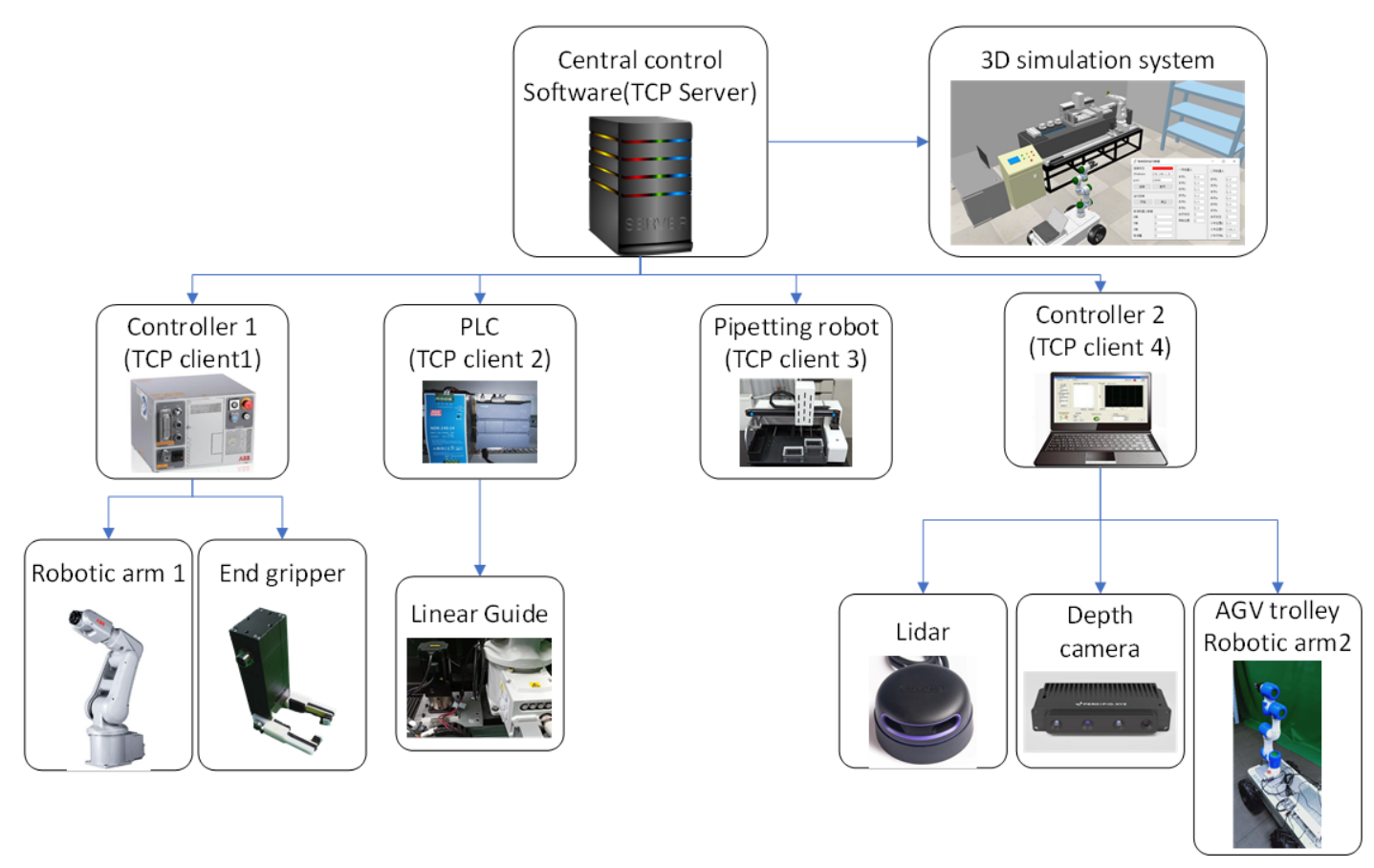

Fig. 5 Communication and control structure of the robotic chemical experiment system.

\section{Fidelity of the Robotic Chemical Operation}

As formaldehyde is a common pollutant in food and water and detrimental to public health, the first model was chosen as the condensation reaction between formaldehyde and 2,4-dinitrobenzene hydrazine (DNPH). Because of the robustness and good chemoselectivity of this reaction, the derivation of formaldehyde to 2,4dinitrophenylhydrazone is a classical method for detecting and measuring formaldehyde in biological and environmental samples. ${ }^{21-23}$ The reaction could be carried out in acidic aqueous media with broad concentration in various mixtures giving the same product. As illustrated in Fig. 6 and video file in supporting 
information, four reactions charged with the solution of formaldehyde and DNPH of different molar ratio by robotic or manual pipette was conducted in parallel for 3 runs. Elevated temperature was applied to minimize the difference of operation time used by robot/personnel at room temperature. The samples were analyzed on HPLC instantly after a one-hour reaction (See Table S4-S6, Fig. S10 and S11 in the Supporting Information). The concentrations of formaldehyde 4 stages were plotted against the HPLC area of DNPH derived hydrazone.

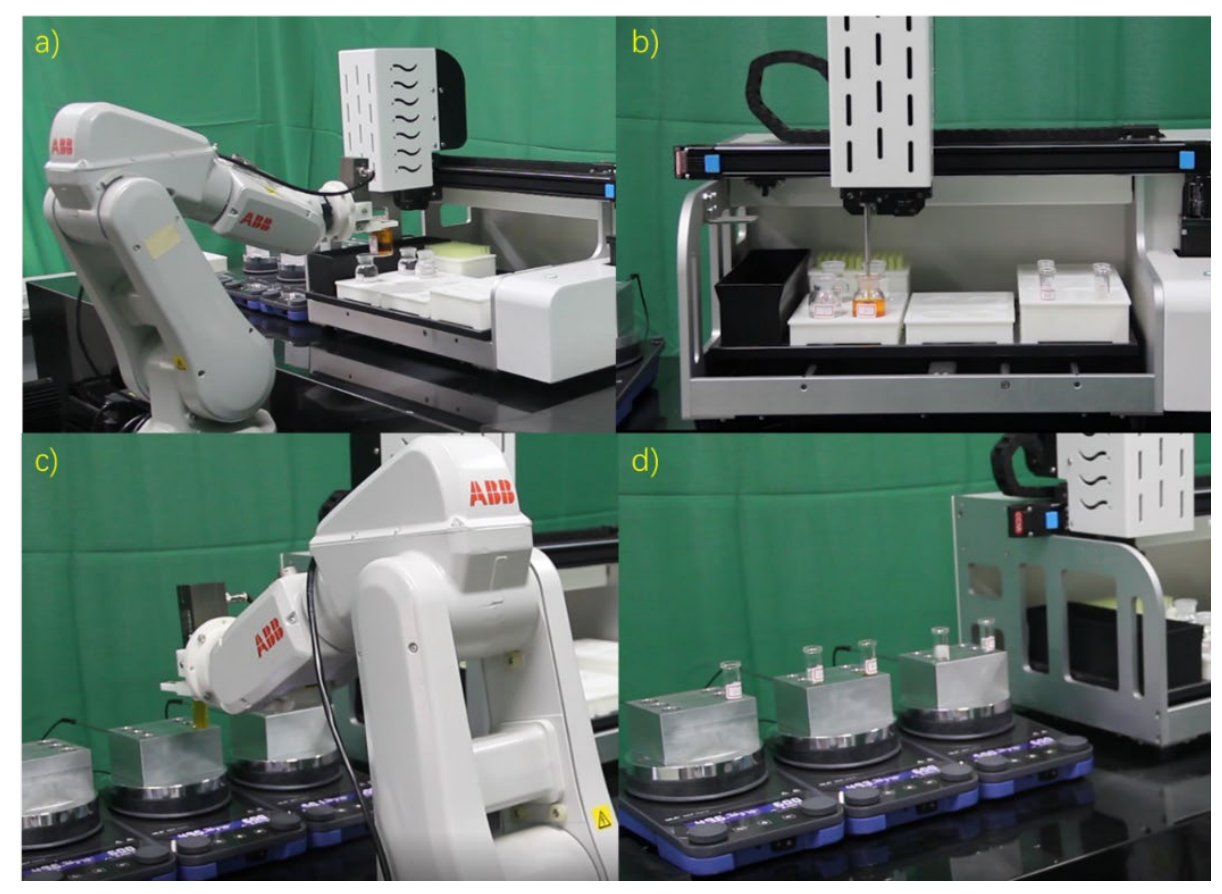

Fig. 6 Robotic chemical operation: a) Moving the reagent bottles and reaction tubes to the pipetting workstation. b) Pipetting. c) Moving the reaction tubes to the Heating/Stirring module. d) Reaction ongoing

The linear regression results obtained from robot and personnel were compared in Fig. 7. It was found both manual and robot operation could give linear results in the range of formaldehyde applied in reaction, suggesting the good reproducibility of robotic operation. Meanwhile, the goodness of fit in three robotic sets (mean 0.99857 , standard deviation 0.00174 ) shows that the repeatability of robotic operation on the automation operation is better than manual operation (mean 0.98667 , standard deviation 0.00443 ). These results suggest that the designed system is reliable for the transfer of experimentation from manual operation 
to robotic ways. The fidelity of robotic chemical operations on this model reaction also shows the potential of this automatic platform in high-throughput formaldehyde determination.
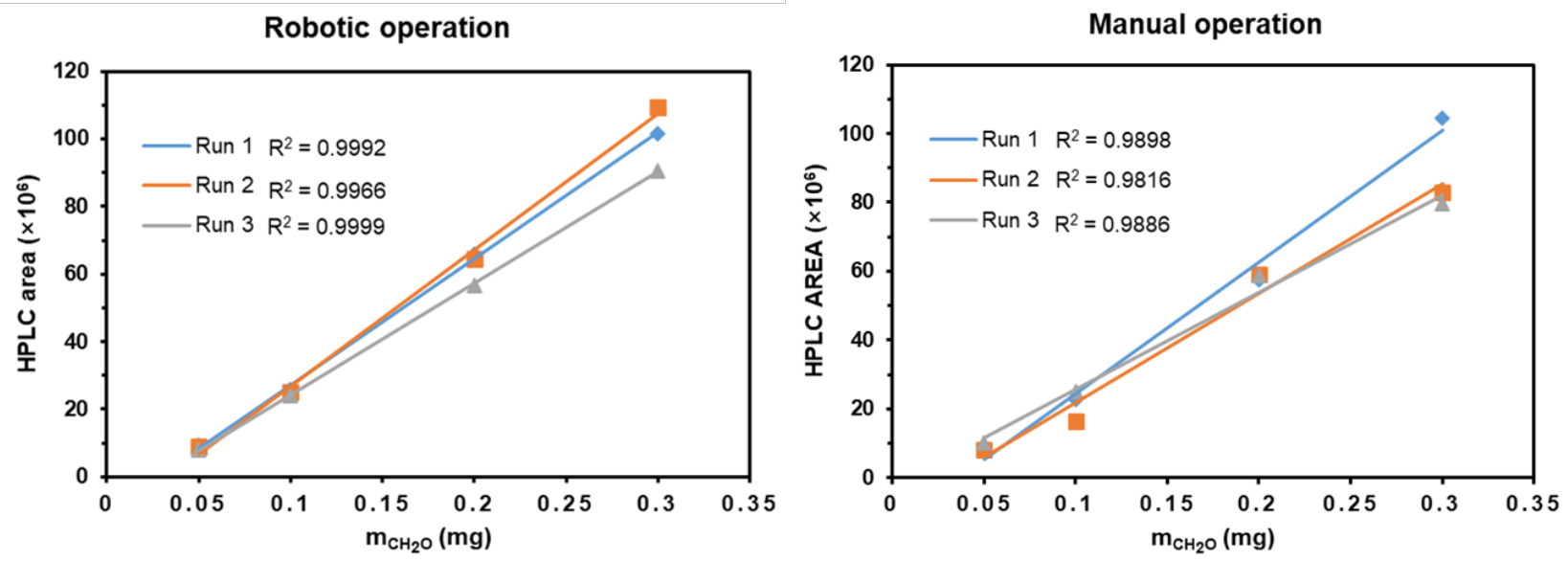

Fig. 7 Comparison of results from robotic/manual chemical operation robotic/manual chemical operation on the reaction of 2,4-dinitrophenyldrazine (DNPH) and $\mathrm{CH}_{2} \mathrm{O}$.

We further explore the ability of the system to simulate reaction condition optimization of a known chemical process, we selected a classical aza Diels-Alder reaction based on a precedent from Kobayashi and coworkers. $^{24}$ In addition to being a powerful strategy to synthesize the drug-like nitrogen-containing heterocycles, ${ }^{28}$ another feature of this reaction is its ecological and low-cost advantages that benefit from being performed in "green" water as reaction media. ${ }^{35,36}$ Because of the insolubility of reactants and products in water, this process provides an opportunity to test the performance of our platform in heterogeneous reactions. As shown in Fig. 7a, five sodium salts were tested for the reaction of Danishefsky's diene with imine 4 in water conducted on three parallels. The stock solution of a catalyst $(0.6 \mathrm{~mL}, 0.05 \mathrm{M})$ and Danishefsky's diene 5 were successively added to the reaction vial with $53.5 \mathrm{mg}$ pre-weighed $\mathrm{N}$ benzylideneaniline 4 . The performance of each catalyst was determined based on the product/internal standard with benzyl ether as an internal standard. The results from the human and robotic operation are collected in Fig.7b. Although the imine and Danishefsky's diene are insoluble in water, this three-phase 
reaction system proceeds rapidly when NaOTf, Nal or 1-dodecanesulfonic acid sodium salt (SDS) was used as the catalysts. However, the reaction is less efficient when performed with $\mathrm{PhSO}_{3} \mathrm{Na}$ or $\mathrm{NaOAc}$ as the catalysts, and only a slight amount of or no desired product was detected. These results indicate that the counterion of the sodium salt has a significant influence on this reaction, which is consistent with previous studies by Kobayashi et al. ${ }^{24}$ Compared with mannual operation, the robotic operated process exhibits good reproducibility with low standard deviations ranging from 0.006 to 0.0036 (see Table S7 and S8 in the Supporting Information). The good consistency of robotic and manual operation demonstrated the potential utility of this platform in the automated reaction condition optimizations.

(a)

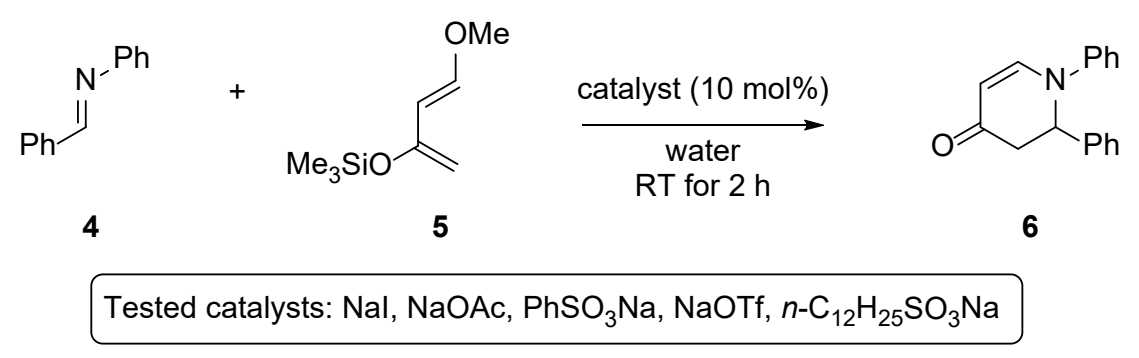

(b)

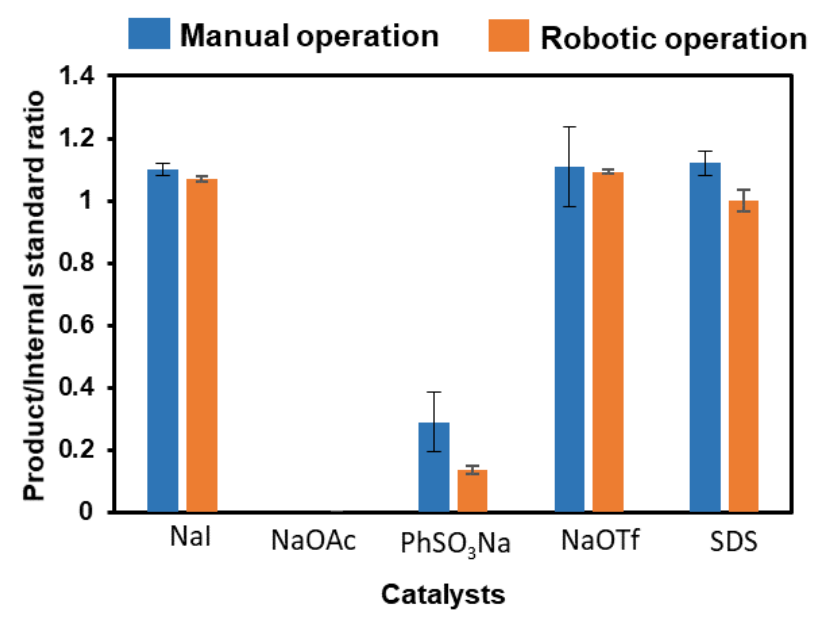

Fig.8 Comparison of manual and robotic catalyst screening for a heterogeneous Aza Diels-Alder reaction: (a) Model reaction; (b) Results of manual and robotic operation for 5 tested sodium salts. All data are presented as mean values $\pm S D$. 


\section{Interface with quantum chemical calculation}

Finally, DFT calculations were conducted to shed light on the mechanistic details for the reaction between 2,4-dinitrophenyldrazine (DNPH) and formaldehyde, with the computational results displayed in Fig. 9. Under acidic condition, the condensation reaction between 2,4-dinitrophenyldrazine and formaldehyde proceeds through two steps: 1) the nucleophilic addition of DNPH affords an intermolecular hydrogen bond-stabilized acetic acid-hemiaminal complex int1 (DNPH $+\mathrm{CH}_{2} \mathrm{O} \rightarrow$ int1); 2 ) dehydration of the hemiaminal intermediate forms the corresponding hydrazone product (int1 $\rightarrow$ pro). Our calculations show that the rate-determining step of this reaction is the dehydration of the DNPH derived hemiamial intermediate int2, and the activation barrier of this step is only about $17 \mathrm{kcal} / \mathrm{mol}$ with the assistance of one molecule of acetic acid. These computational results are consistent with the mild experimental condition, the rapid reaction rate for the condensation reaction of 2,4-dinitrophenyldrazine (DNPH) and formaldehyde. The further hybridation of the present automatic robot operation with high throughput quantum chemical computations ${ }^{37-38}$ and the automatic reaction pathway searching strategy such as $M D / C D^{39-40}$ would build a flatform the automatic rational design of chemical reactions and catalysts from the first principle. 


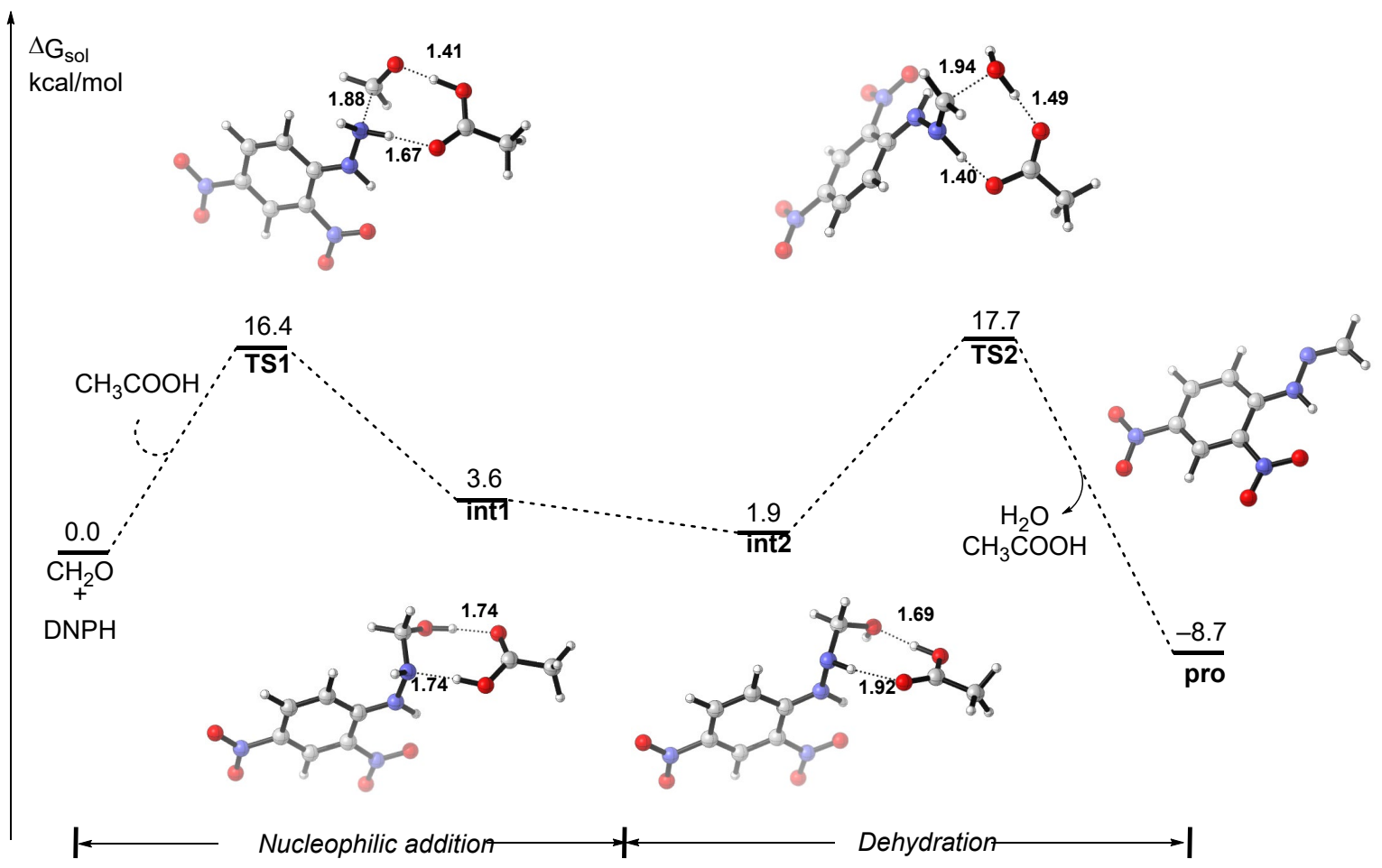

Fig. 9 Gibbs free energy profile for the reaction of 2,4-dinitrophenyldrazine (DNPH) with $\mathrm{CH}_{2} \mathrm{O}$. Performed at B3LYP-D3/Def2-TZVP(PCM, $\left.\mathrm{H}_{2} \mathrm{O}\right)$ // B3LYP-D3/Def2-SVP (PCM, $\left.\mathrm{H}_{2} \mathrm{O}\right)$ level of theory. Color code: white H; C, gray; $\mathrm{N}$, blue; $\mathrm{O}$, red.

\section{Conclusion}

In summary, we have developed a highly reliable, flexible and general automatic chemical reaction machine by the use of a coordinated multi-robot system. Fidelity test on two model reactions demonstrates that this platform is a promising tool for the automation of chemical synthesis including both homogenous and heterogeneous reactions. Given the capability of $24 / 7$ working and applicability to different types of reactions, the use of this automatic chemical reaction machine for future high-throughput reaction screening ${ }^{41,42}$ is in progress in our laboratory. The combination of the automatic reaction machine with computational-based automatic reaction design too ${ }^{39,40}$ is highly desired to develop an intelligent reaction system integrating reaction design, chemical synthesis, data analysis and feedback, leading to the upgrading 
of the research paradigm of synthetic chemistry from "labor-intensive and trial and error experiment" to "automation and intelligence".

\section{Supporting Information}

Supporting Information is available and includes complete descriptions of experimental procedures, calculations, structures, and a video for the robotic operation.

\section{Conflict of Interest (required)}

There is no conflict of interest to report.

\section{Funding Information (required)}

This work was supported by the National Key Research and Development Program of China (Grant Number 2019YFC0408303), National Natural Science Foundation of China (Grant Number 62073160, 22033004).

\section{Preprint Acknowledgement (required, if applicable)}

Research presented in this article was posted on a preprint server prior to publication in CCS Chemistry. The corresponding preprint article can be found here: (DOI; Direct Link)

\section{Acknowledgements (required, if applicable)}

The authors would like to thank Mr. Buyin Li, Mr. Huiqiao Fu, Mr. Qixin Zhou and Mr. Jiaxu Bai for helping to implement and performing the robotic systems.

\section{References}

1. Merrifield, R. B.; Stewart, J. M.; Jernberg, N., Instrument for Automated Synthesis of Peptides. Anal. Chem. 1966, 38, 1905-1914.

2. Dimitrov, T.; Kreisbeck, C.; Becker, J. S.; Aspuru-Guzik, A.; Saikin, S. K., Autonomous Molecular Design: Then and Now. ACS Appl. Mater. Interfaces 2019, 11, 24825-24836.

3. Granda, J. M.; Donina, L.; Dragone, V.; Long, D.-L.; Cronin, L., Controlling an organic synthesis robot with machine learning to search for new reactivity. Nature 2018, 559, 377-381.

4. Dragone, V.; Sans, V.; Henson, A. B.; Granda, J. M.; Cronin, L., An autonomous organic reaction search engine for chemical reactivity. Nat. Commun. 2017, 8, 15733. 
5. Steiner, S.; Wolf, J.; Glatzel, S.; Andreou, A.; Granda, J. M.; Keenan, G.; Hinkley, T.; Aragon-Camarasa, G.; Kitson, P. J.; Angelone, D.; Cronin, L., Organic synthesis in a modular robotic system driven by a chemical programming language. Science 2019, 363, eaav2211.

6. Li, J.; Ballmer, S. G.; Gillis, E. P.; Fujii, S.; Schmidt, M. J.; Palazzolo, A. M. E.; Lehmann, J. W.; Morehouse, G. F.; Burke, M. D., Synthesis of many different types of organic small molecules using one automated process. Science 2015, 347, 1221-1226.

7. Fleischer, H.; Baumann, D.; Joshi, S.; Chu, X.; Roddelkopf, T.; Klos, M.; Thurow, K., Analytical Measurements and Efficient Process Generation Using a Dual-Arm Robot Equipped with Electronic Pipettes. Energies 2018, 11, 2567.

8. Fleischer, H.; Thurow, K., On the Way to Efficient Analytical Measurements: The Future of Robot-Based Measurements. SLAS Technol.: Translating Life Sciences Innovation 2020, 25, 208-211.

9. Grainger, R.; Whibley, S., A Perspective on the Analytical Challenges Encountered in High-Throughput Experimentation. Org. Process Res. Dev. 2021, 25, 354-364.

10. Li, Z.; Najeeb, M. A.; Alves, L.; Sherman, A. Z.; Shekar, V.; Parrilla, P. C.; Pendleton, I. M.; Wang, W.; Nega, P. W.; Zeller, M.; Schrier, J.; Norquist, A. J.; Chan, E. M., Robot-Accelerated Perovskite Investigation and Discovery. Chem. Mater. 2020, 32, 5650-5663.

11. Tabor, D. P.; Roch, L. M.; Saikin, S. K.; Kreisbeck, C.; Sheberla, D.; Montoya, J. H.; Dwaraknath, S.; Aykol, M.; Ortiz, C.; Tribukait, H.; Amador-Bedolla, C.; Brabec, C. J.; Maruyama, B.; Persson, K. A.; Aspuru-Guzik, A., Accelerating the discovery of materials for clean energy in the era of smart automation. Nat. Rev. Mater. 2018, 3, 520.

12. Epps, R. W.; Bowen, M. S.; Volk, A. A.; Abdel-Latif, K.; Han, S.; Reyes, K. G.; Amassian, A.; Abolhasani, M., Artificial Chemist: An Autonomous Quantum Dot Synthesis Bot. Adv. Mater. 2020, 32, 2001626.

13. Bedard, A.-C.; Adamo, A.; Aroh, K. C.; Russell, M. G.; Bedermann, A. A.; Torosian, J.; Yue, B.; Jensen, K. F.; Jamison, T. F., Reconfigurable system for automated optimization of diverse chemical reactions. Science 2018, 361, 1220-1225.

14. Hardwick, T.; Ahmed, N., Digitising chemical synthesis in automated and robotic flow. Chem. Sci. 2020, 11, 11973-11988.

15. Chatterjee, S.; Guidi, M.; Seeberger, P. H.; Gilmore, K., Automated radial synthesis of organic molecules. Nature 2020, 579, 379-384.

16. Trobe, M.; Burke, M. D., The Molecular Industrial Revolution: Automated Synthesis of Small Molecules. Angew. Chem. Int. Ed., 2018, 57, 4192-4214.

17. Burger, B.; Maffettone, P. M.; Gusev, V. V.; Aitchison, C. M.; Bai, Y.; Wang, X.; Li, X.; Alston, B. M.; Li, B.; Clowes, R.; Rankin, N.; Harris, B.; Sprick, R. S.; Cooper, A. I., A mobile robotic chemist. Nature 2020, 583, $237-241$.

18. Ibarz, J. T., J.; Finn, C.; Kalakrishnan, M.; Pastor, P.; and Levine, S., How to train your robot with deep reinforcement learning: lessons we have learned. Int. J. Rob. Res. 2021, DOI: 10.1177/0278364920987859.

19. Dogar, M.; Spielberg, A.; Baker, S.; Rus, D., Multi-robot grasp planning for sequential assembly operations. Auton. Robots. 2019, 43, 649-664.

20. Arulkumaran, K.; Deisenroth, M. P.; Brundage, M.; Bharath, A. A., Deep Reinforcement Learning A brief survey. IEEE Signal Proc. Mag. 2017, 34, 26-38.

21. Lipari, F.; Swarin, S. J., Determination of Formaldehyde and Other Aldehydes in Automobile Exhaust with an Improved 2,4-Dinitrophenylhydrazine Method. J. Chromatogr. 1982, 247, 297-306.

22. Pardakhti, A. R.; Bidhendi, G. R. N.; Namdari, R., Determination of formaldehyde in environmental and other aqueous samples. Asian. J. Chem. 2008, 20, 849-852.

23. Wahed, P.; Razzaq, M. A.; Dharmapuri, S.; Corrales, M., Determination of formaldehyde in food and feed by an in-house validated HPLC method. Food Chem. 2016, 202, 476-483.

24. Loncaric, C.; Manabe, K.; Kobayashi, S., Alkaline salt-catalyzed aza Diels-Alder reactions of Danishefsky's diene with imines in water under neutral conditions. Chem. Commun. 2003, 574-575.

25. Hwangbo, J.; Lee, J.; Dosovitskiy, A.; Bellicoso, D.; Tsounis, V.; Koltun, V.; Hutter, M., Learning agile and dynamic motor skills for legged robots. Sci. Robot. 2019, 4, eaau5872.

26. Mnih, V.; Kavukcuoglu, K.; Silver, D.; Rusu, A. A.; Veness, J.; Bellemare, M. G.; Graves, A.; Riedmiller, M.; Fidjeland, A. K.; Ostrovski, G.; Petersen, S.; Beattie, C.; Sadik, A.; Antonoglou, I.; King, H.; Kumaran, D.; Wierstra, D.; 
Legg, S.; Hassabis, D., Human-level control through deep reinforcement learning. Nature 2015, 518, 529-33.

27. Lee, C.; Yang, W.; Parr, R. G., Development of the Colle-Salvetti correlation-energy formula into a functional of the electron density. Phys. Rev., B Condens. Matter. 1988, 37, 785-789.

28. Grimme, S.; Antony, J.; Ehrlich, S.; Krieg, H., A consistent and accurate ab initio parametrization of density functional dispersion correction (DFT-D) for the 94 elements H-Pu. J. Chem. Phys. 2010, 132, 154104.

29. Grimme, S.; Hansen, A.; Brandenburg, J. G.; Bannwarth, C., Dispersion-Corrected Mean-Field Electronic Structure Methods. Chem. Rev. 2016, 116, 5105-5154.

30. Weigend, F.; Ahlrichs, R., Balanced basis sets of split valence, triple zeta valence and quadruple zeta valence quality for $\mathrm{H}$ to $\mathrm{Rn}$ : Design and assessment of accuracy. Phys. Chem. Chem. Phys. 2005, 7, 3297-3305.

31. Becke, A. D., Density-functional thermochemistry. III. The role of exact exchange. J. Chem. Phys. 1993, 98, 5648-5652.

32. Tomasi, J.; Persico, M., Molecular-Interactions in Solution-an Overview of Methods Based on Continuous Distributions of the Solvent. Chem. Rev. 1994, 94, 2027-2094.

33. Tao, F.; Zhan, H.; Liu, A.; Nee, A. Y. C., Digital Twin in Industry: State-of-the-Art. IEEE Trans. Ind. Appl. 2019, 15, 2405-2415.

34. Kal-Koshvandi, A. T.; Heravi, M. M., Applications of Dainshefsky's Dienes in the Asymmetric synthesis of Aza-Diels-Alder Reaction. Chem. Rec. 2019, 19, 550-600.

35. Chanda, A.; Fokin, V. V., Organic Synthesis "On Water". Chem. Rev. 2009, 109, 725-748.

36. Candeias, N. R.; Branco, L. C.; Gois, P. M. P.; Afonso, C. A. M.; Trindade, A. F., More Sustainable Approaches for the Synthesis of N-Based Heterocycles. Chem. Rev. 2009, 109, 2703-2802.

37. Jain, A.; Hautier, G.; Moore, C. J.; Ong, S. P.; Fischer, C. C.; Mueller, T.; Persson, K. A.; Ceder, G., A highthroughput infrastructure for density functional theory calculations. Comput. Mater. Sci. 2011, 50, $2295-2310$.

38. Chung, Y. G.; Camp, J.; Haranczyk, M.; Sikora, B .J.; Bury, W.; Krungleviciute, V.; Yildirim, T.; Farha, O. K.; Sholl, D. S.; Snurr, R. Q., Computation-Ready, Experimental Metal-Organic Frameworks: A Tool To Enable HighThroughput Screening of Nanoporous Crystals. Chem. Mater. 2014, 26, 6185-6192.

39. Yang, M.; Zou, J.; Wang, G.; Li, S., Automatic Reaction Pathway Search via Combined Molecular Dynamics and Coordinate Driving Method. J. Phys. Chem. A 2017, 121, 1351-1361.

40. Yang, M.; Yang, L.; Wang, G.; Zhou, Y.; Xie, D.; Li, S., Combined Molecular Dynamics and Coordinate Driving Method for Automatic Reaction Pathway Search of Reactions in Solution. J. Chem. Theory. Comput. 2018, 14, 57875796.

41. Krska, S. W.; DiRocco, D. A.; Dreher, S. D.; Shevlin, M., The Evolution of Chemical High-Throughput Experimentation To Address Challenging Problems in Pharmaceutical Synthesis. Acc. Chem. Res. 2017, 50, 29762985.

42. Shevlin, M., Practical High-Throughput Experimentation for Chemists. ACS. Med. Chem. Lett. 2017, 8, 601607. 
Table of Contents Graphic (required)

Place Table of Contents the graphic here.
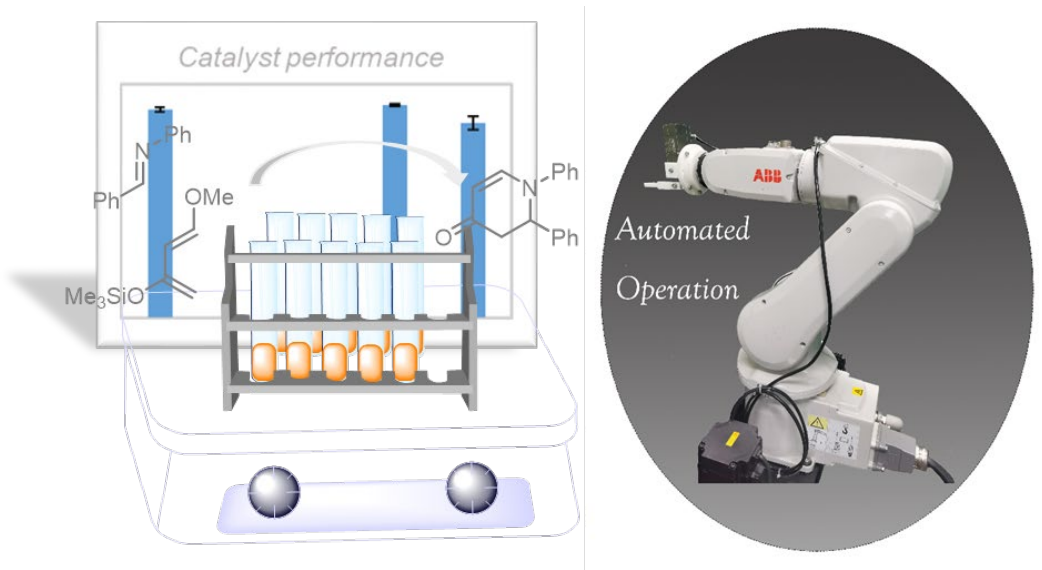